\title{
Three-Dimensional 1060-nm OCT: Choroidal Thickness Maps in Normal Subjects and Improved Posterior Segment Visualization in Cataract Patients
}

\author{
Marieh Esmaeelpour, ${ }^{1}$ Boris Považay, ${ }^{1,2}$ Boris Hermann, ${ }^{1,2}$ Bernd Hofer, ${ }^{1,2}$ Vedran Kajic, ${ }^{1}$ \\ Ketan Kapoor, ${ }^{1}$ Nik J. L. Sheen, ${ }^{1}$ Rachel V. North, ${ }^{1}$ and Wolfgang Drexler ${ }^{1,2}$
}

Purpose. To evaluate the performance and potential clinical role of three-dimensional (3D) 1060-nm OCT by generating choroidal thickness (ChT) maps in patients of different ages with different degrees of ametropia and axial lengths and to investigate the effect of cataract grade on OCT retinal imaging quality.

Methods. Axial lengths (ALs) and $45^{\circ}$ fundus photographs were acquired from 64 eyes (34 healthy subjects, 19 to 80 years, ametropia +3 to -10 D). 3D 1060-nm OCT was performed over a $36^{\circ} \times 36^{\circ}$ field of view with $\sim 7-\mu \mathrm{m}$ axial resolution and up to 70 frames/s (512 A-scans/frame). ChT maps between retinal pigment epithelium and the choroidal-scleral interface, were generated and statistically analyzed. A further 30 eyes (19 subjects), with cataracts assessed with the LOCS III scale, were imaged with 3D 1060-nm OCT and 800-nm OCT, and visualization of the posterior segment was compared qualitatively.

Results. In 64 eyes, ChT maps displayed a thickness decrease with increasing AL. Subfoveal ChT was $315 \pm 106 \mu \mathrm{m}$ (mean \pm $\mathrm{SD})$, negatively correlated with $\mathrm{AL}\left(R^{2}=-0.47, P<0.001\right)$. Averaged ChT maps of eyes with $\mathrm{AL}<23.39 \mathrm{~mm}$ showed an increased ChT in an area $\sim 1500 \mu \mathrm{m}$ inferior, compared with subfoveal ChT. Eyes with $\mathrm{AL}>24.5 \mathrm{~mm}$ showed a larger variation and a thicker ChT superiorly than inferiorly. Reduced signal strength in cataractous eyes was found in $65 \%$ of the 800 -nm OCT images, but in only $10 \%$ of the 1060 -nm OCT images.

Conclusions. The imaging performance of 3D 1060-nm OCT is unique, producing maps that show the variation in ChT over the entire field of view, in relation to axial length. This imaging system has the potential of visualizing a novel clinical diagnostic biomarker. Compared with 800 -nm OCT, it provides superior visualization of the posterior pole in cataractous eyes. (Invest Ophthalmol Vis Sci. 2010;51:5260-5266) DOI: 10.1167/iovs. 10-5196

From the ${ }^{1}$ School of Optometry and Vision Sciences, Cardiff University, Cardiff, United Kingdom; and the ${ }^{2}$ Center for Medical Physics and Biomedical Engineering, Medical University of Vienna, Vienna, Austria.

Supported in part by the BBSRC (Biotechnology and Biological Sciences Research Council); Cardiff University FP6-IST-NMP-2 Strept (017128, NanoUB); a DTI (Department of Trade and Industry) grant (OMICRON); an AMR (Action Medical Research) Grant AP1110; European Union project FunOCT FP7 Health Contract 201880; Carl Zeiss Meditec Inc.; and Diabetes UK Grant BDA:RD07/003472.

Submitted for publication January 12, 2010; revised March 16, 2010; accepted March 28, 2010.

Disclosure: M. Esmaeelpour, None; B. Považay, None; B. Hermann, None; B. Hofer, None; V. Kajic, None; K. Kapoor, None; N.J.L. Sheen, None; R.V. North, None; w. Drexler, Carl Zeiss Meditec (C)

Corresponding author: Wolfgang Drexler, Medical University Vienna, Center for Medical Physics and Biomedical Engineering, General Hospital Vienna, 4L, Waehringer Guertel 18-20, A-1090, Vienna, Austria; wolfgang.drexler@meduniwien.ac.at.
$\mathrm{H}$ igh-resolution, high-speed, three-dimensional optical coherence tomography at $1060 \mathrm{~nm}$ (3D 1060-nm OCT) images the retina and, in contrast to OCT at $800 \mathrm{~nm}$, significantly increases choroidal penetration, ${ }^{1-6}$ with less light-scattering by cataract. ${ }^{2}$ Previously, in vivo investigation of the choroid has been possible with ultrasound and MRI, but with limited resolution. ${ }^{7,8}$ Histology of choroidal thickness (ChT) reveals increased ChT that correlates with a higher density of vessels in the superficial choroidal layers in open-angle glaucoma, ${ }^{9}$ atrophy of the choroidal capillary structure, ${ }^{10}$ and neovascularization in the choriocapillaris. ${ }^{11,12}$ Histologic studies investigated biological structures with comparable high resolution, but these were limited by preparation artifacts, shrinkage, and lack of blood supply to sustain vasculature volume. ${ }^{13}$ Commercial OCT systems operating at $800 \mathrm{~nm}$ have shown choroidal penetration only in thin choroids of myopic subjects, or by imaging limited to two-dimensional (2D) horizontal scans. ${ }^{14-16}$ The clinical value of imaging the choroid has been shown in a small sample of patients, by using OCT at $1060 \mathrm{~nm}$ to demonstrate ChT variation over the field of view in uveal inflammation ${ }^{17}$ and to demonstrate an increase in vessel density at the center of choroidal neovascularization in age-related macular degeneration (ARMD). ${ }^{2,18}$ Recent interest in the choroid led to a study in which 3D 1060-nm OCT was used to investigate ChT confined to central and four peripheral measurement locations in a healthy Japanese population. ${ }^{19}$ However, analyzing the measurements at several image locations neglects the remaining data collected over the entire imaged field. ChT changes may occur in patients with diabetes or ARMD, and both these groups have a high incidence of cataract. With the current commercial OCT systems that work at $800 \mathrm{~nm}$, cataract may reduce imaging quality or make image acquisition unsuccessful. ${ }^{20}$ In contrast to OCT at $800 \mathrm{~nm}$, light is less scattered by cataract when OCT at $1060 \mathrm{~nm}$ is used, ${ }^{2}$ but it is not clear whether visualizing the ChT with a 3D 1060-nm OCT can be successful in eyes with dense cataract.

In two separate studies, we explored the performance of OCT at $1060 \mathrm{~nm}$, to penetrate the highly light-scattering choroid. The first study of choroidal visualization performance was conducted to quantify the complete 3D 1060-nm OCT scan with ChT maps and to investigate the ChT maps in healthy subjects across a wide range of ametropia and axial eye length (AL). The second is a study of 3D 1060-nm OCT performance in the presence of dense cataracts to determine retinal and choroidal visibility where the $800-\mathrm{nm}$ OCT fails.

\section{Methods}

\section{Subjects for ChT Maps}

Thirty-four healthy male and female subjects between the ages of 19 and 80 years participated (Table 1). Exclusion criteria were a history of retinal surgery, retinal disease; physiological variation from normal, 
Table 1. Demographic and Statistical Data of the Cohort

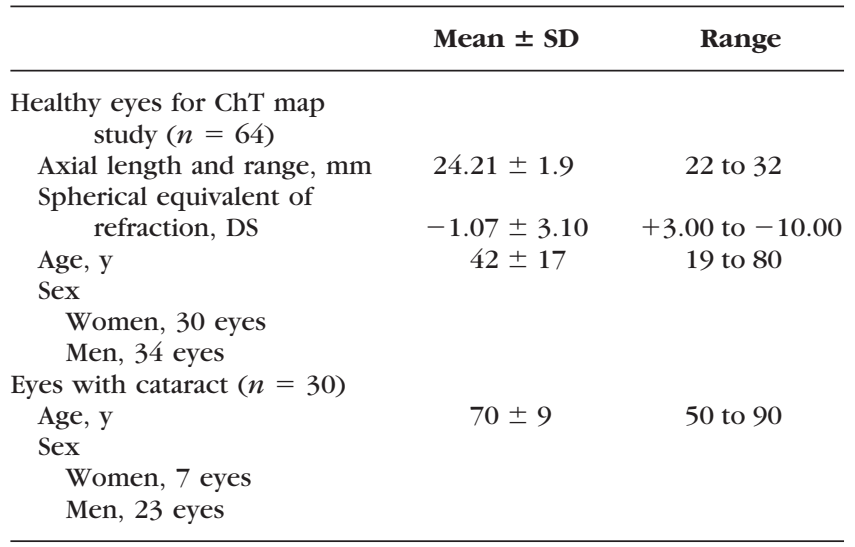

such as drusen, epiretinal membranes, or central retinal degeneration; or systemic disease, such as diabetes mellitus. After the application of these criteria, 64 eyes were included in the choroidal study. Monocular visual acuity was determined with the ETDRS Original Series Charts (Precision Vision, La Salle, IL). All subjects had visual acuity ranging between -0.1 and $+0.3 \operatorname{logMAR}$, and all imaging was conducted without pupil dilation. The refractive error was measured with an autorefractometer (KR-7500; Topcon, Tokyo, Japan).

\section{OCT Imaging in Cataractous Eyes}

Thirty eyes of 19 subjects (Table 1 ) with cataract were graded in vivo at the slit lamp, according to the Lens Opacities Classification System III (LOCS III). ${ }^{21}$ LOCS III categorizes lens opacification into nuclear color (NC), nuclear opacity (NO), cortical cataract (C), and posterior subcapsular cataract $(\mathrm{P})$. It applies photographic standards to grade cataract severity on a decimal scale between 0.1 and 6 units for NC and NO, and 0.1 and 5.9 units for $\mathrm{C}$ and $\mathrm{P}$. In this study, exclusion criteria were pseudoaphakia and any cataract with an LOCS III scale of $\leq 1$ unit. Cataractous eyes with opacification in multiple classifications were chosen to evaluate the consistency of image quality in a wide range of overall cataract density, regardless of classification.

Ethics approval was obtained from the School of Optometry and Vision Sciences, Cardiff University Ethics Committee, and the Central and Local Office for Research Ethics Committees. The studies adhered to the Declaration of Helsinki, and informed consent was obtained from all subjects before participation.

\section{OCT Imaging and Ocular Biometry for ChT Maps}

Retinal status was evaluated with a fundus photograph with a $45^{\circ}$ field centered on the macula, taken with a nonmydriatic retinal camera (CR-DGi; Canon, Inc. Lake Success, NY). Five AL (cornea-RPE) measurements were averaged from each eye by optical biometry (IOL Master; Carl Zeiss Meditec, Jena, Germany). High-speed 3D OCT-imaging at $1060 \mathrm{~nm}$ was performed with less than $2.5 \mathrm{~mW}$ at the cornea, well below the maximum power limit for a 10-second exposure. ${ }^{22,23}$ Three-dimensional (3D) OCT volumes were acquired at $1060 \mathrm{~nm}$ with 15 to $20 \mu \mathrm{m}$ transverse resolution, $\sim 7 \mu \mathrm{m}$ axial resolution, and 512 voxels per depth scan (A-scan). Scans across a $36^{\circ} \times 36^{\circ}$ field and an $18^{\circ} \times 18^{\circ}$ field with $512 \times 512 \mathrm{~A}$-scans at 47,000 A-scans/s were centered on the fovea and produced up to 70 frames/s. For image processing and manual measurement of pixel distances in the choroid and retina, ImageJ software ${ }^{24}$ was used.

\section{OCT Imaging for the Cataract Eyes}

Subjects were imaged with 1060-nm OCT as described previously, all eyes were additionally imaged with an 800-nm OCT (Cirrus HD-OCT; Carl Zeiss Meditec). System specifications of both OCT instruments
(800 $\mathrm{nm}$ and $1060 \mathrm{~nm}$ ), such as $\sim 97-\mathrm{dB}$ signal-to-noise ratio and their roll-off with scanning depth of $-6 \mathrm{~dB}$ at $\sim 1.1 \mathrm{~mm}, \sim 6-$ to $7-\mu \mathrm{m}$ axial resolution, and $2.6-\mathrm{mm}$ scanning depth were comparable and thus allowed an objective and fair comparison of the wavelength effect on posterior pole visualization performance. Imaging was performed over a field of view of $20^{\circ}$ centered around the fovea with $512 \times 512$ A-scans and 128 B-scans.

\section{Data Analysis for ChT Maps}

For ChT maps, a field of $36^{\circ} \times 36^{\circ}$ scans with each degree corresponding to $\sim 288 \mu \mathrm{m},{ }^{25}$ was centered on the fovea. The OCT volume was averaged in both transverse directions within a field of $\sim 1^{\circ}$, to remove speckle and increase sensitivity. Axial ChT was defined as the distance between the center of the peaks originating from the RPE/Bruch's membrane/choriocapillaris (RBC) $\operatorname{complex}^{26}$ and the choroid-sclera interface (CSI). For the investigation of the ChT variation throughout the entire field of view, thickness maps were generated based on manual segmentation, with segmentation in every fourth tomogram at the RBC complex and the CSI. Two-dimensional ChT maps, generated by local subtraction of the segmented height profiles were smoothed by transverse filtering and splining between the slices, followed by a final $2 \mathrm{D}$ median filter with a $15 \times 15 \mathrm{kernel}$ size. The resulting pixel
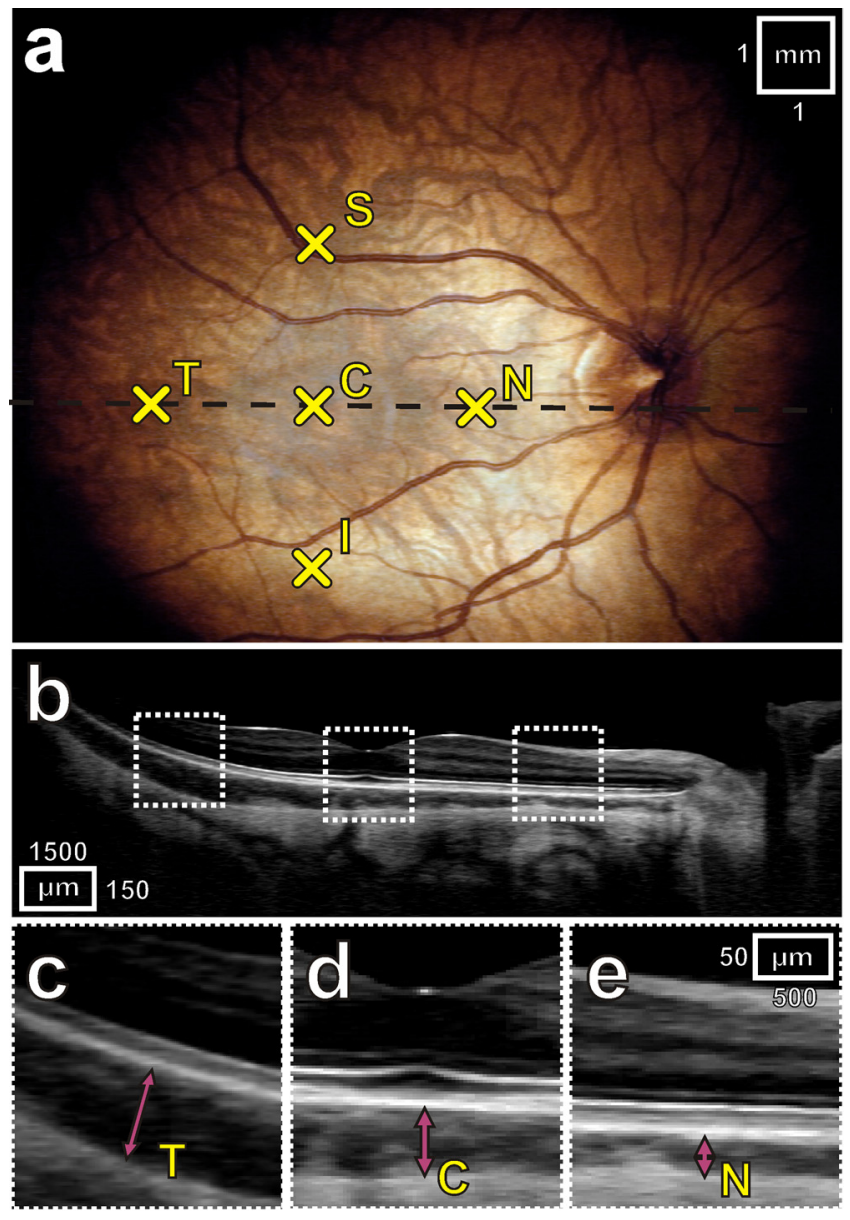

FIGURE 1. Images generated from the 1060-nm OCT stack. (a) En face view (generated by integrated OCT 3D stack in depth) depicting the $\mathrm{ONH}$ and the fovea. $\times$, locations of the thickness measurements: central (C), superior (S), nasal (N), inferior (I), temporal (T). (b) Overview of a central cross-section indicates the locations of the $\mathrm{T}$, $\mathrm{C}$, and $\mathrm{N}$ measurement distances. Dashed lines: sections magnified in (c, d, e); pink arrows: ChT measurement. 
distance was converted into optical distance by using the depth sampling calibration for the 1060-nm OCT system and to physiological distance by using a group refractive index of $n=1.4$ (based on the refractive index of blood). This method resulted in thickness maps for individual eyes. For statistical analysis of the mean and variation of the ChT maps of individuals with similar ametropia, all the maps were aligned with one another with respect to the macula and optic nerve position (MatLab software; The MathWorks, Inc., Natick, MA). After correcting for differences in transverse area size with $\mathrm{AL}$, an intensity overlay was introduced to exclude unreliable portions of the images with less than five measurements at one location. The images were evaluated along the depth-scan direction. ChT maps were grouped by judging the eyes' refraction to be myopic (AL $\geq 24.5 \mathrm{~mm} ; n=16$ ), emmetropic ( $24.5>\mathrm{AL} \geq 23.4 \mathrm{~mm}, n=20$ ), or hyperopic ( $\mathrm{AL}<23.4$ $\mathrm{mm} ; n=28$ ), on the basis of normal AL variation with refraction and age in the literature. ${ }^{27-29}$ Groups are described as characteristically myopic (long ALs), emmetropic (mid length eyes), and hyperopic (short eyes). Mean and SD was obtained to create a compound map of average thickness for these three groups of eyes with color-coded thickness.

For statistical assessment of individual variation between eyes, central ChT was located for the choroid beneath the fovea. Peripheral ChT was determined for the nasal, temporal, superior, and inferior choroid at a distance of $7.5^{\circ}$ to the foveal center (Fig. 1) Statistical testing for normally distributed data and adjustments so that outliers did not affect the statistical outcomes were performed. Multiple regression analysis was performed to assess the influence of ametropia, $\mathrm{AL}$, age, and sex on ChT at five different choroid locations (SPSS 16; SPSS, Inc., Chicago, IL).

\section{Data Analysis for Images in Cataractous Eyes}

The same processing protocol was used for images of the choroid taken by both OCT devices in the cataract study. To keep the comparison of the en face views similar, we did not include the imaged choroid when generating en face images averaged between the RPE and the ILM. One investigator, blind to the type of OCT used, assessed imaging performance in tomograms acquired with both wavelengths by searching for noticeable changes in contrast, localized signal strength decrease, blurring within en face views, and tomograms of eyes with cataracts and compared it to that in images of eyes without cataract in the healthy study group.

\section{Results}

\section{ChT Maps}

For the 64 healthy eyes, all tomograms were of sufficient quality to allow ChT measurement. Although the eyes were not dilated, image signal in the $36^{\circ} \times 36^{\circ}$ scans was acquired in a field of at least $30^{\circ}$.

ChT maps (Fig. 2) show mean ChT distribution and the coefficient of variation over the imaged field of view. Hyperopic eyes had a central ChT of $358 \pm 96 \mu \mathrm{m}$ (mean \pm SD) and had the thickest choroid inferiorly but still within a $1500-\mu \mathrm{m}$ distance from the center. Emmetropic eyes had a ChT of $341 \pm$ $95 \mu \mathrm{m}$, whereas myopic eyes had the thinnest choroid of the three groups, with a central ChT of $213 \pm 58 \mu \mathrm{m}(P<0.001$, ANOVA, Bonferroni post hoc testing) and an increase of thickness approximately $1500 \mu \mathrm{m}$ superior to the center. For illustrating the location and extent of variation in the data sets, we found the coefficient of variation useful in making comparisons of widely different means. The coefficient of variation is expressed as a percentage of the standard deviation from the mean. The coefficient of variation was lowest in hyperopic eyes, less than $30 \%$ over most of the ChT map. In emmetropes, the areas that had this low variation were smaller, and in myopes the predominant coefficient of variation was between $30 \%$ and $45 \%$.

Results of the regression analysis for each of the three groups showed that only the ChT in myopes correlated significantly with age and $\mathrm{AL}$, depending on the location measured (Table 2). In emmetropic eyes, the ChT variation could not be statistically explained by the factors that we examined. The temporal ChT in hyperopic eyes weakly but significantly correlated with $\mathrm{AL}$. For all eyes, the mean $\pm \mathrm{SD}$ of $\mathrm{ChTs}$ were $315 \pm 106 \mu \mathrm{m}(\mathrm{C}), 250 \pm 113 \mu \mathrm{m}(\mathrm{N}), 276 \pm 95 \mu \mathrm{m}(\mathrm{T})$, $315 \pm 112 \mu \mathrm{m}$ (S), and $293 \pm 106 \mu \mathrm{m}$ (I). ALs correlated negatively with ChTs for all five transverse locations (Fig. 3; Table 2). Regression analysis of all 64 eyes found no relationship for age and sex $(P>0.05)$. The spherical equivalent of the prescription also correlated with ChT $\left(R^{2}=-0.29 P<0.05\right)$, with hyperopic eyes having the thickest and myopic eyes the thinnest choroid. However, since AL and ametropia are two correlating factors, the refractive error was excluded because of its colinearity with AL.
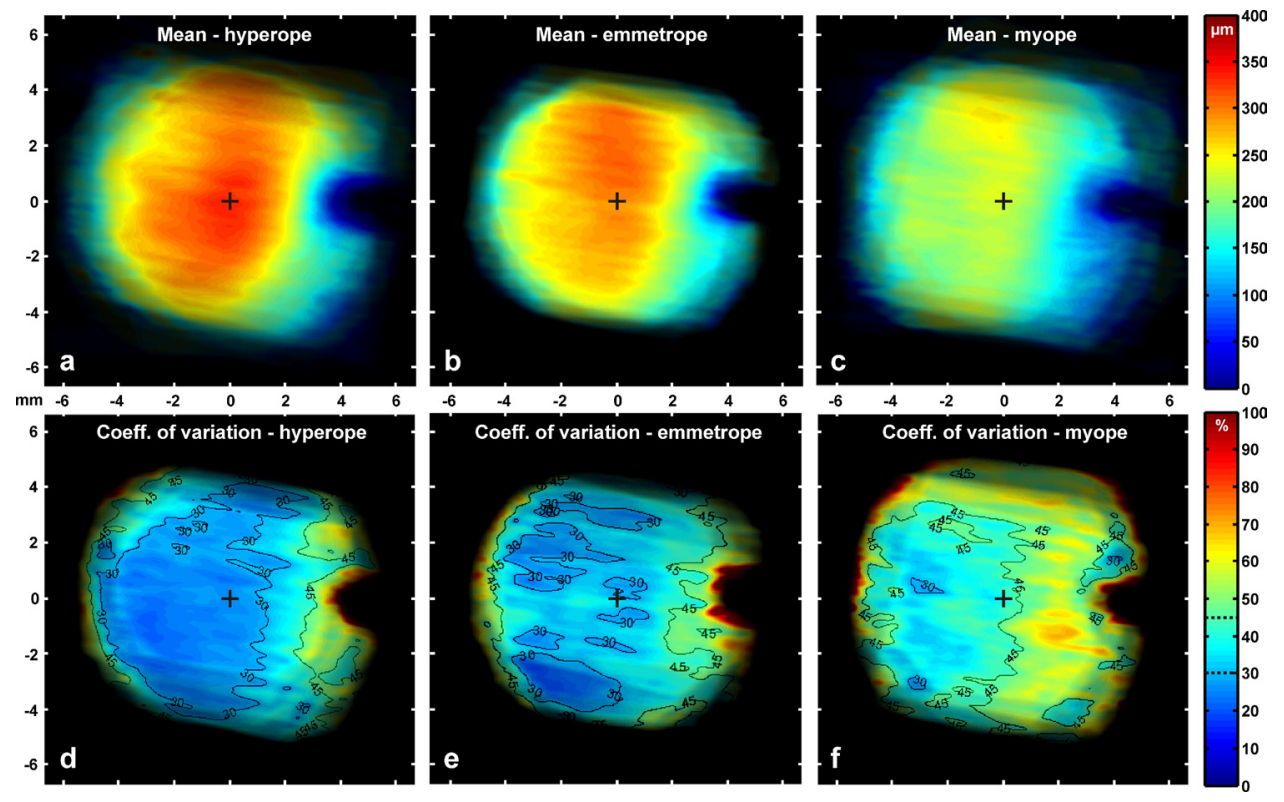

Figure 2. ChT maps, grouped by axial length, to distinguish the degree of ametropia (hyperopic, short eyes: $\mathrm{AL}<23.4 \mathrm{~mm}$; emmetropic midlength eyes: $24.5 \mathrm{~mm}>\mathrm{AL} \geq 23.4 \mathrm{~mm}$ and myopic long eyes: $\mathrm{AL} \geq 24.5 \mathrm{~mm}$ ). + , the fovea. (a-c) Mean ChT was highest in hyperopes, and its distribution throughout the posterior eye differed between groups. (d-f) Coefficients of variation showed areas of variation below $30 \%$ to be largest in the hyperope group and to decrease in myopes. 
TABLE 2. Results of Statistical Testing of Choroidal Thickness Variation by Multiple Regression Analysis

\begin{tabular}{|c|c|c|c|c|c|c|c|c|}
\hline & \multicolumn{2}{|c|}{$\begin{array}{l}\text { All Eyes } \\
(n=64)\end{array}$} & \multicolumn{2}{|c|}{$\begin{array}{l}\text { Hyperopic Eyes } \\
(n=28)\end{array}$} & \multicolumn{2}{|c|}{$\begin{array}{c}\text { Emmetropic Eyes } \\
(n=20)\end{array}$} & \multicolumn{2}{|c|}{$\begin{array}{l}\text { Myopic Eyes } \\
(n=16)\end{array}$} \\
\hline & Factor & $R^{2}$ & Factor & $R^{2}$ & Factor & $R^{2}$ & Factor & $R^{2}$ \\
\hline Central & $\mathrm{AL}$ & $-0.43^{*}$ & $\neq$ & 0.12 & $\neq$ & 0.21 & AL, age & $-0.76 \dagger$ \\
\hline Nasal & $\mathrm{AL}$ & $-0.35^{*}$ & $\ddagger$ & 0.17 & $\ddagger$ & 0.1 & $\mathrm{AL}$ & $-0.55 \dagger$ \\
\hline Temporal & $\mathrm{AL}$ & $-0.41^{*}$ & $\mathrm{AL}$ & $-0.23 \dagger$ & $\ddagger$ & 0.27 & $\mathrm{AL}$ & $-0.55 \dagger$ \\
\hline Superior & $\mathrm{AL}$ & $-0.44^{*}$ & $\neq$ & 0.18 & $\neq$ & 0.21 & $\mathrm{AL}$ & $-0.55 \dagger$ \\
\hline Inferior & $\mathrm{AL}$ & $-0.44^{*}$ & $\neq$ & 0.04 & $\ddagger$ & 0.1 & AL, age & $-0.85 \dagger$ \\
\hline
\end{tabular}

The factors axial eye length (AL), age, and sex were examined. First column: locations where ChT was measured. Column headings: groups of eyes that were examined in the analysis. Only cells with significant models (significant $R^{2}$ value) contain the contributing factor(s).

$* P<0.001$.

$\dagger P<0.05$.

$\ddagger$ No factor contributed to the model.

\section{D 1060-nm OCT Performance in Cataract Eyes}

For the subjects with cataracts, the mean visual acuity was $0.22 \pm 0.21 \mathrm{log}$ units. LOCS classification and grades for all eyes and eyes grouped by OCT device and image quality are shown in Table 3. In 30 eyes, cataract image quality was reduced in $13(65 \%)$ imaged at $800 \mathrm{~nm}$ and in $3(10 \%)$ imaged at $1060 \mathrm{~nm}$. The decreased image quality was observed as local reduced signal strength that appeared like a shadow with diffuse borders within the retina extending into the choroid. Figure 4 shows representative OCT images of eyes with a range of cataracts from low to high grade. The shadow width and intensity in the 3D 1060-nm OCT images were smaller than in the $800-\mathrm{nm}$ OCT images, and it was not located in the same region.

In the 1060-nm OCT scans with good signal strength, the retina, choroid, and the choroid interface with the sclera were visible. There was no overall signal reduction in eyes with dense cataracts (median of 3.5 units). With the 800-nm OCT, the eyes with grades $\mathrm{NO}>3, \mathrm{NC}>4, \mathrm{C}>2.5$, and $\mathrm{P}>4$ were imaged with a reduced signal strength. The three 1060-nm OCT images with reduced signal strength had LOCS III grades of $\mathrm{NO} 5, \mathrm{NC6}, \mathrm{C} 5$, and $\mathrm{P} 3$; $\mathrm{NO} 3, \mathrm{NC} 5, \mathrm{C} 3$, and $\mathrm{P} 5$; and $\mathrm{NO} 2$, $\mathrm{NC} 4, \mathrm{C} 3$, and $\mathrm{P} 4$.

The visual acuity of the subject with the densest cataract (Fig. 5, subject 30), which had reduced signal strength on both OCT instruments, was $+0.34 \log$ units. The visual acuity of the other two subjects with reduced signal strength on the 3D 1060-nm OCT images were $+0.5 \mathrm{log}$ units (Fig. 5, subject 23) and $+0.3 \log$ units (Fig. 5, subject 21).

\section{Discussion}

In the present study, 3D 1060-nm OCT visualized the entire depth of the choroid in an area larger than $30^{\circ}$ in all imaged eyes and without pupil dilation. Subsequent mapping of ChT showed individual variation in ChT distribution among subjects, and, when grouped, the variation was strongest on the basis of AL. In individuals with mild, moderate, and dense
Figure 3. ChT in five different locations versus axial eye length. Trendlines describe the negative statistical relationship for all locations.

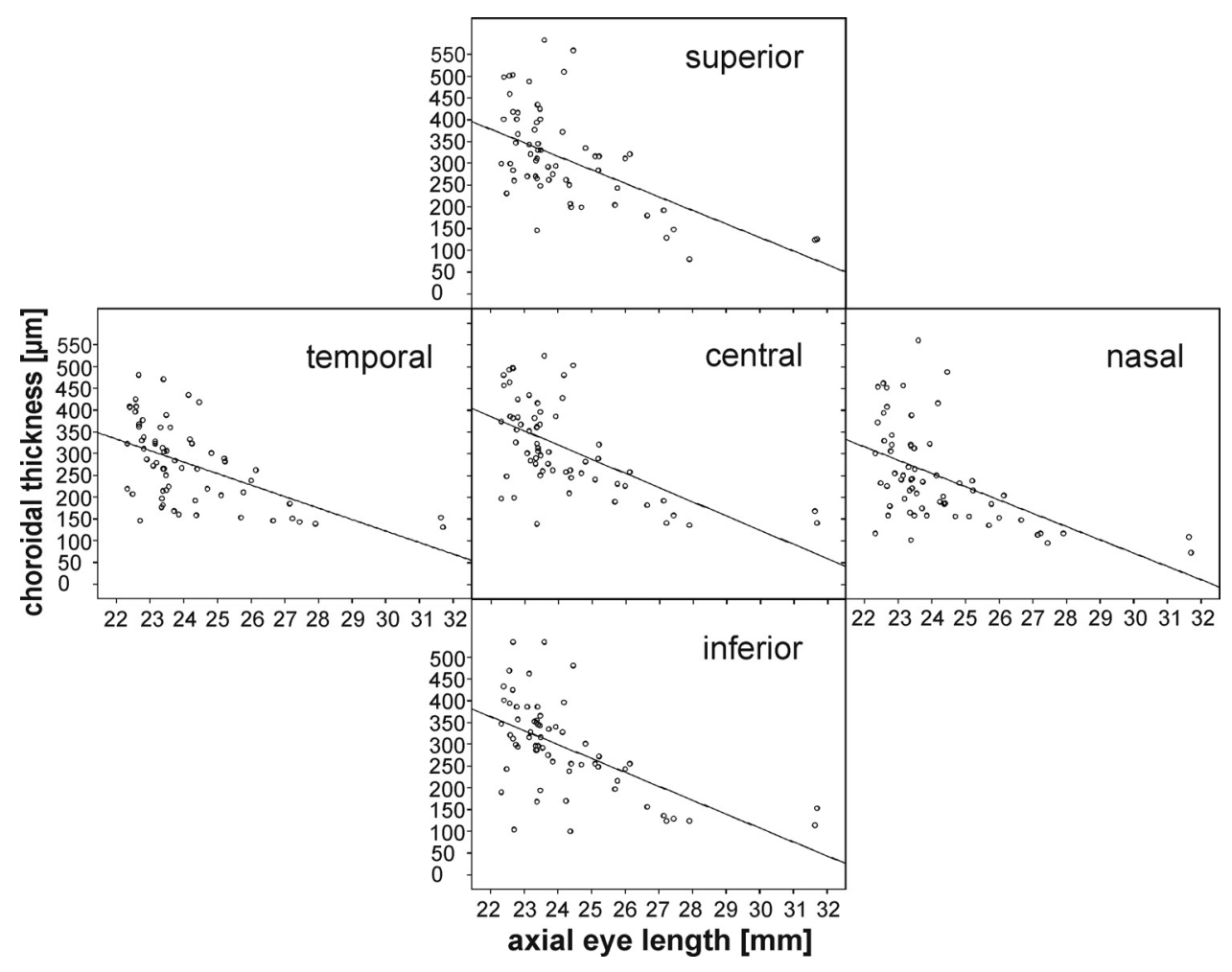


TABLE 3. LOCS III Classification of Cataract Images with Both Devices

\begin{tabular}{ccccc}
\hline & & \multicolumn{2}{c}{$\mathbf{8 0 0 - n m ~ O C T}$} \\
\cline { 3 - 4 } $\begin{array}{c}\text { LOCS III } \\
\text { Classification }\end{array}$ & $\begin{array}{c}\text { All Eyes } \\
(\boldsymbol{n}=\mathbf{3 0 )}\end{array}$ & $\begin{array}{c}\text { Good Signal Strength } \\
(\boldsymbol{n}=\mathbf{1 7})\end{array}$ & $\begin{array}{c}\text { Reduced Signal Strength } \\
(\boldsymbol{n}=\mathbf{1 3})\end{array}$ & $\begin{array}{c}\text { 3D 1060-nm-OCT Good Signal } \\
\text { Strength }(\boldsymbol{n}=\mathbf{2 7})\end{array}$ \\
\hline NO & $3(1-5)$ & $3(1-3)$ & $3(2-5)$ & $3(1-5)$ \\
NC & $3.5(2-6)$ & $3(2-4)$ & $4(3-6)$ & $3.5(2-5)$ \\
C & $2(1-5)$ & $2(1-2.5)$ & $3(2-5)$ & $2(1-5)$ \\
P & $3(1-5)$ & $2(1-4)$ & $3.5(1-5)$ & $2(1-5)$ \\
\hline
\end{tabular}

Data are expressed as the median (range).

cataracts, the 3D 1060-nm OCT was superior to an 800-nm system in obtaining posterior pole images.

To date, wide area measurement of ChT with OCT at 800 $\mathrm{nm}$ has been conducted in myopic eyes ${ }^{14,16}$ with the subfoveal choroid in these studies over a similar sized area being much thinner than that in this study $\left(100.5 \pm 56.9 \mu \mathrm{m}^{14}\right.$ and $93.2 \pm$ $62.5 \mu \mathrm{m}^{16}$ ). This difference is explainable by the inclusion of patients with myopia of $\geq 6 \mathrm{D}$. The distribution at peripheral choroidal positions was similar to that in the present study, with the superior choroid being the thickest of all measurements. However, results similar to those in the present study have been found with high-resolution ultrasound imaging with a mean ChT of $320 \mu \mathrm{m}$ in healthy eyes ${ }^{7}$ and, with 3D 1060-nm OCT, of $354 \pm 111 \mu \mathrm{m}$ in Japanese subjects. ${ }^{19}$ In the latter study, ${ }^{19}$ ChT was measured at five locations, and the inferior choroid was thinner than the superior choroid. This finding is in agreement with the mean of the representative ChT locations in all eyes in the present study, but a large individual variation was observed. A correlation of central and inferior ChT variation with age was found only in the group of longer, myopic eyes. Instead, the overall ChT was characterized by AL. Of all biometric parameters, AL correlates strongest with the spherical equivalent and contributes to the other biometric parameters of the eye. ${ }^{30}$ As well as a negative correlation of ChT with higher ALs, a large ChT variation was displayed in the coefficient of variation map of the longer, myopic eyes, perhaps because of the inclusion of a larger range of ALs in the data for the longer eyes, and may also be attributable to the myopic eye shape. A study with a wide-field, high-resolution scanning photorefractor, a system designed for the measurement of the peripheral refraction, found that eye shape in moderate myopia presents with more irregularity than in emmetropia. ${ }^{31}$ The investigators hypothesized that this was due to changes in the sclera and therefore was not detectable with a commercial OCT. The sclera may be weaker in myopes with a correlation between high myopia and scleral thinning found in human eyes. ${ }^{32}$ The choroid adheres to the sclera because of the fixed position of the blood vessels passing through the sclera, and it is likely that the choroid is stretched and thins after scleral changes in myopic eyes. ${ }^{33}$ Therefore, the present study suggests ChT maps for a detailed investigation of the entire field of view, although manual segmentation is sufficiently time consuming to make its use in a clinical setting difficult at present.

The present study revealed a decrease in ChT with age at the central and inferior locations in eyes with the longer ALs
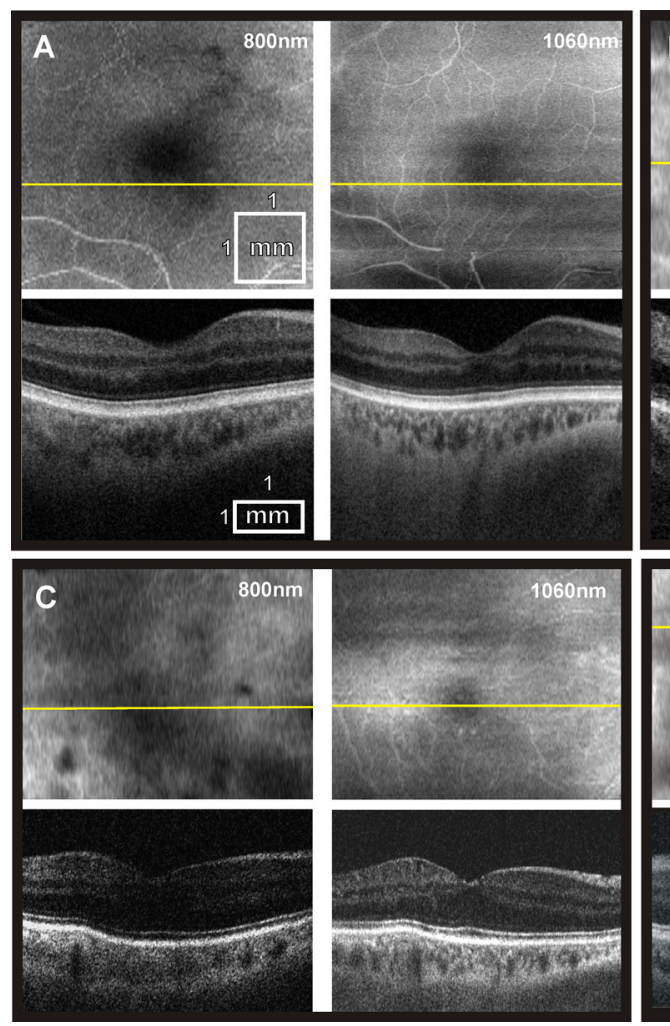

Figure 4. En face view and tomograms generated from 3D 1060-nm OCT and 800 -nm OCT stacks from four subjects with an increasing degree of cataract and loss of image signal strength: (A) NC1, NO2, C1.7, and P1; (B) NC3, NO3, C2, and P1; (C) NC4, NO4.5, C5, and P4; and (D) NC5, NO6, C5, and P3. 


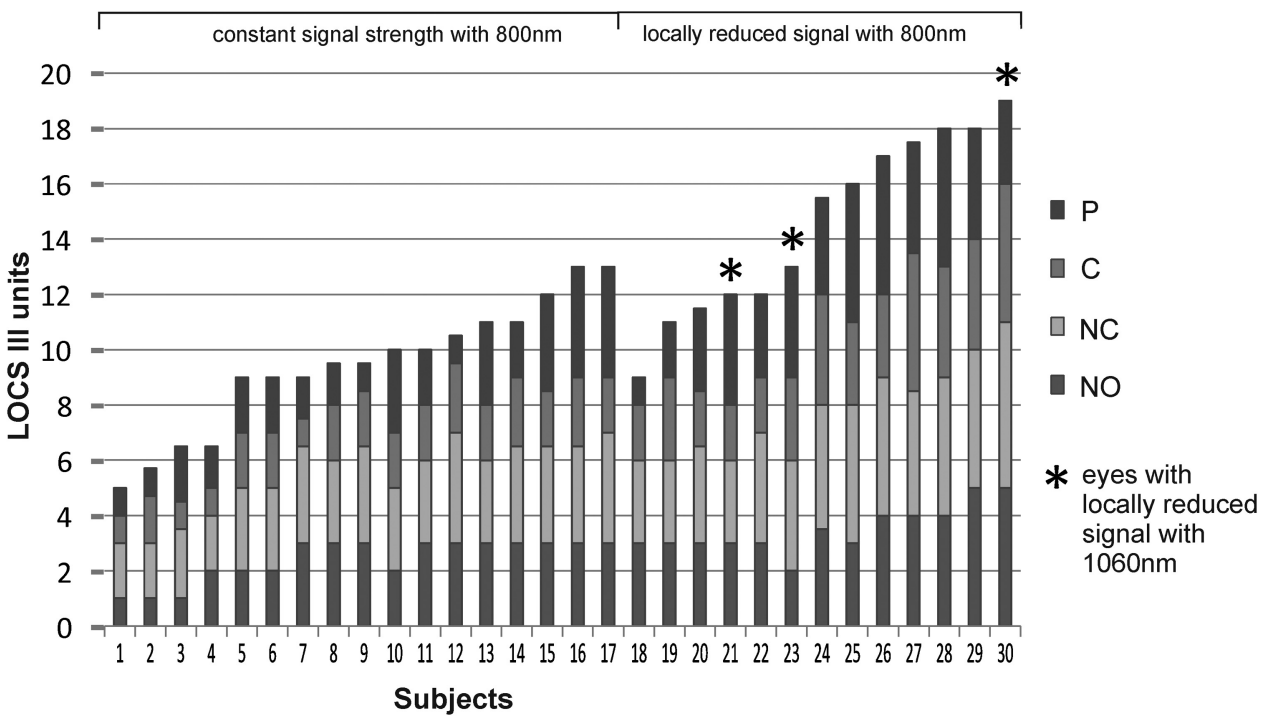

FIGURE 5. Signal quality in relation to cataract density in retinal and choroidal images taken with 800 - and 1060-nm OCTs
Subjects typically associated with myopia, which is in agreement with the findings of other investigators. ${ }^{15,19}$ In contrast to these results, it has been shown that with advancing age, the choroid in mice thickens and morphologic age changes suggest choroidal vascular inflammation with age. ${ }^{34}$ Visualization of ChT and structure will be of special interest in the study of diseases with suspected choroidal inflammation. At a molecular level, choroidal inflammation has become the recent focus of investigation. Documentation of VAP-1, induced at sites of inflammation, ${ }^{35}$ is found in both the retina and choroid arterial vessels of the human eye. ${ }^{36}$ A sensitive, noninvasive in vivo imaging device for investigating the structural change is useful for inflammatory diseases affecting ocular vasculature, such as diabetes, rheumatoid arthritis, and atherosclerosis. ${ }^{37}$ Experimental findings of ocular inflammation demonstrate involvement of all choroid layers. However, with 3D 1060-nm OCT, the choriocapillaris is not discernible, as it is below the axial resolution of the present $1060-\mathrm{nm}$ instrument. ${ }^{38}$

Cataract affects image quality by reducing the signal-tonoise ratio and therefore the signal strength of OCT at $800 \mathrm{~nm}$ because of to light-scattering, and at present the only way to improve the ratio is by removing the cataract. ${ }^{20}$ To our knowledge, no other study has investigated the imaging performance of 3D 1060-nm OCT in the posterior pole in patients with mild to severe cataracts or compared it to the quality achieved with 800-nm OCT. In a study of 800-nm OCT, only moderate cataracts were included in evaluating OCT signal strengths. ${ }^{20}$ In a previous study with moderate cataracts in which OCT at 800 $\mathrm{nm}$ failed, OCT at $1060 \mathrm{~nm}$ provided detail of the retinal and choroidal structures irrespective of the opaque cataractous lens. ${ }^{2}$ With a large range of cataract grades, image quality at $800 \mathrm{~nm}$ decreased with moderate to dense cataracts. The 1060 nm-3D-OCT acquired good-quality images with constant signal strength in 27 eyes compared with only 17 with images of good quality acquired by an 800-nm OCT. The en face visualization of the stacks showed that areas of reduced signal strength were smaller and did not overlap in 3D 1060-nm OCT tomograms compared with the 800-nm OCT (Fig. 4, subject D). Visualization of the posterior eye is crucial to patients' clinical management, especially in the presence of diabetes or ARMD. Some participants in this study had secondary dense cataracts due to other disease. Retinal health could not be monitored accurately with any other technique, and cataracts were not removed before the patient's participation, because it was not clear whether the patient would benefit. The results of this study showed that the performance of 3D 1060-nm OCT can be clinically useful in certain cases and that the visualization of ChT was not affected by cataract density. It is possible that extraneous factors, such as pupil margin artifacts or unstable subject fixation, precluded the 3D 1060-nm OCT from obtaining good-quality images with no reduction in signal strength in a few of the cases. The LOCSIII does not specify the exact location of cortical and posterior cataracts. The variation in the spatial location of the cataract may explain why there was no consistency in image quality and signal strength with the same LOCS III grades.

In conclusion, this study establishes ChT distribution by mapping, justifying the transverse resolution and visualization of a large field of view of the 3D 1060-nm OCT with a continuous measurement coverage. 3D high-speed 1060-nm OCT enables retina and choroid visualization and choroidal quantification, resulting in ChT maps and a superior imaging ability in dense cataracts, which cannot be achieved with any other currently commercially available OCT technique. The literature shows potential involvement of the choroid in myopia, ageing, and ocular diseases, but this notion has not been sufficiently researched in vivo because of the lack of a commercial high-resolution, 3D imaging device with choroid and cataract penetration. Future 3D 1060-nm OCT studies of the choroid's characteristics in ocular abnormalities or diseases should include an examination of axial length.

\section{Acknowledgments}

The authors thank James Morgan, Sarah Hale, and Jez Guggenheim for valuable support throughout the study.

\section{References}

1. Unterhuber A, Považay B, Hermann B, Sattmann H, Chavez-Pirson A, Drexler W. In vivo retinal optical coherence tomography at $1040 \mathrm{~nm}$-enhanced penetration into the choroid. Optics Express. 2005;13(9):3252-3258.

2. Považay B, Hermann B, Unterhuber A, et al. Three-dimensional optical coherence tomography at $1050 \mathrm{~nm}$ versus $800 \mathrm{~nm}$ in retinal pathologies: enhanced performance and choroidal penetration in cataract patients. $J$ Biomed Opt. 2007;12(4):7.

3. Lee EC, de Boer JF, Mujat M, Lim H, Yun SH. In vivo optical frequency domain imaging of the human retina and choroid. $O p t$ Express. 2006;14(10):4403-4411.

4. Považay B, Hermann B, Hofer B, et al. Wide field optical coherence tomography of the choroid in vivo. Invest Opbthalmol Vis Sci. 2008:1856-1863. 
5. Yasuno Y, Miura M, Kawana K, et al. Visualization of sub-retinal pigment epithelium morphologies of exudative macular diseases by high-penetration optical coherence tomography. Invest $O p h$ thalmol Vis Sci. 2009;50(1):405-413.

6. Makita S, Fabritius T, Yasuno Y. Full-range, high-speed, high-resolution 1-micron spectral-domain optical coherence tomography using BM-scan for volumetric imaging of the human posterior eye. Opt Express. 2008;16(12):8406-8420.

7. Coleman DJ, Silverman RH, Chabi A, et al. High-resolution ultrasonic imaging of the posterior segment. Opbthalmology. 2004; 111(7): $1344-1351$.

8. Townsend KA, Wollstein G, Schuman JS. Clinical application of MRI in ophthalmology. NMR Biomed. 2008;21(9):997-1002.

9. Yin ZQ, Vaegan, Millar TJ, Beaumont P, Sarks S. Widespread choroidal insufficiency in primary open-angle glaucoma. J Glaucoma. 1997;6(1):23-32.

10. McLeod DS, Lutty GA. High-resolution histologic analysis of the human choroidal vasculature. Invest Opbthalmol Vis Sci. 1994; 35(11):3799-3811.

11. Sarks SH. Ageing and degeneration in the macular region: a clinicopathological study. Br J Ophthalmol. 1976;60(5):324-341.

12. Green WR, Key SN 3rd. Senile macular degeneration: a histopathologic study. Trans Am Opbthalmol Soc. 1977;75:180-254.

13. Gloesmann M, Hermann B, Schubert C, Sattmann H, Ahnelt PK, Drexler W. Histologic correlation of pig retina radial stratification with ultrahigh-resolution optical coherence tomography. Invest Ophthalmol Vis Sci. 2003;44(4):1696-16703.

14. Ikuno Y, Tano Y. Retinal and choroidal biometry in highly myopic eyes using spectral-domain optical coherence tomography. Invest Ophthalmol Vis Sci. 2009;50(8):3876-3880.

15. Margolis R, Spaide RF. A pilot study of enhanced depth imaging optical coherence tomography of the choroid in normal eyes. Am J Opbthalmol. 2009;147(5):811-815.

16. Fujiwara T, Imamura Y, Margolis R, Slakter JS, Spaide RF. Enhanced depth imaging optical coherence tomography of the choroid in highly myopic eyes. Am J Opbthalmol. 2009;148(3):445-450.

17. Yasuno Y, Okamoto F, Kawana K, Yatagai T, Oshika T. Investigation of multifocal choroiditis with panuveitis by three-dimensional high-penetration optical coherence tomography. $J$ Biopbotonics. 2009;2(6-7):435-441.

18. de Bruin DM, Burnes DL, Loewenstein $\mathrm{J}$, et al. In vivo threedimensional imaging of neovascular age-related macular degeneration using optical frequency domain imaging at $1050 \mathrm{~nm}$. Invest Opbthalmol Vis Sci. 2008;49(10):4545-4552.

19. Ikuno Y, Kawaguchi K, Yasuno Y, Nouchi T. Choroidal thickness in healthy Japanese subjects. Invest Opbthalmol Vis Sci. 2010;51: 2173-2176.

20. van Velthoven ME, van der Linden MH, de Smet MD, Faber DJ, Verbraak FD. Influence of cataract on optical coherence tomography image quality and retinal thickness. Br J Ophthalmol. 2006; 90(10):1259-1262.

21. Chylack LT Jr, Wolfe JK, Singer DM, et al. The Lens Opacities Classification System III. The Longitudinal Study of Cataract Study Group. Arch Opbthalmol. 1993;111(6):831-836.
22. American National Standard Institute (ANSI). Safe Use of Lasers and Safe Use of Optical Fiber Communications. New York: American National Standard Institute, Z136 Committee; 2000:168.

23. ICNIRP. Revision of the Guidelines on Limits of Exposure to Laser radiation of wavelengths between $400 \mathrm{~nm}$ and $1.4 \mu \mathrm{m}$. In: Society HP, editor. International Commission on Non-Ionizing Radiation Protection: International Commission on Non-Ionizing Radiation Protection. 2000:431-440.

24. ImageJ. 1.42e-k version. Bethesda, MD: National Institutes of Health; 1997-2009.

25. Drasdo N, Fowler CW. Non-linear projection of the retinal image in a wide-angle schematic eye. Br J Opbthalmol. 1974;58(8):709714 .

26. Anger EM, Unterhuber A, Hermann B, et al. Ultrahigh resolution optical coherence tomography of the monkey fovea: identification of retinal sublayers by correlation with semithin histology sections. Exp Eye Res. 2004;78(6):1117-1125.

27. Llorente L, Barbero S, Cano D, Dorronsoro C, Marcos S. Myopic versus hyperopic eyes: axial length, corneal shape and optical aberrations. J Vis. 2004;4(4):288-298.

28. Lee KE, Klein BE, Klein R, Quandt Z, Wong TY. Association of age, stature, and education with ocular dimensions in an older white population. Arch Ophthalmol. 2009;127(1):88-93.

29. Strang NC, Schmid KL, Carney LG. Hyperopia is predominantly axial in nature. Curr Eye Res. 1998;17(4):380-383.

30. Touzeau O, Allouch C, Borderie V, Kopito R, Laroche L. Correlation between refraction and ocular biometry (in French). $J \mathrm{Fr}$ Opbtalmol. 2003;26(4):355-363.

31. Tabernero J, Schaeffel F. More irregular eye shape in low myopia than in emmetropia. Invest Opbthalmol Vis Sci. 2009;50(9):45164522 .

32. Avetisov ES, Savitskaya NF, Vinetskaya MI, Iomdina EN. A study of biochemical and biomechanical qualities of normal and myopic eye sclera in humans of different age groups. Metab Pediatr Syst Ophthalmol. 1983;7(4):183-188.

33. Wildsoet CF. Myopia and Nearwork. Oxford: Butterworth-Heinemann; 1998:31-90.

34. Xu H, Chen M, Forrester JV. Para-inflammation in the aging retina. Prog Retin Eye Res. 2009;28(5):348-368.

35. Jalkanen S, Salmi M. Vascular adhesion protein-1 (VAP-1): a new adhesion molecule recruiting lymphocytes to sites of inflammation. Res Immunol. 1993;144(9):746-749; discussion 754-762.

36. Almulki L, Noda K, Nakao S, Hisatomi T, Thomas KL, HafeziMoghadam A. Localization of vascular adhesion protein-1 (VAP-1) in the human eye. Exp Eye Res. 2009.

37. O'Sullivan J, Unzeta M, Healy J, O'Sullivan MI, Davey G, Tipton KF. Semicarbazide-sensitive amine oxidases: enzymes with quite a lot to do. Neurotoxicology. 2004;25(1-2):303-315.

38. Torti C, Považay B, Hofer B, et al. Adaptive optics optical coherence tomography at 120,000 depth scans/s for non-invasive cellular phenotyping of the living human retina. Opt Express. 2009;17: 19382-19400. 